\title{
Importance des corps gras dans le domaine des tensioactifs
}

Jérôme GUILBOT

SEPPIC,

127, Chemin de la Poudrerie,

BP 228,

81105 Castres, France,

Fax : +33(0)563726967

$<$ jerome.guilbot@airliquide.com>

\begin{abstract}
This article is willing to show through different industrial examples the role played by the oleochemistry in the development of new surfactants.

This field offers indeed a large choice of alkyl chains that can be transformed. Linked to a polar head such as a polyol, a carbohydrate or a protein derivative, these alkyl chains lead to performing bio-surfactants that show interesting toxicological and ecotoxicological profiles.

This natural lipophilic source allows thus to adjust some physico-chemical parameters of the surfactant such as its geometry, its hydrophilic lipophilic balance, the type of functional groups linking its hydrophilic part to its lipophilic part, its molecular weight, etc. The emulsifying, foaming, solubilizing, biological properties can be valued in cosmetics (as excipients or actives), in the areas of veterinary additives, of detergents, in the food industry, etc.
\end{abstract}

Key words: oleochemistry, natural lipophilic source, hydrophilic lipophilic balance, polyol

tifs « non renouvelables » possédant une partie lipophile et une partie hydrophile issues de la filière pétrochimique. À titre d'exemples, on peut citer les alkyl benzène sulfonates linéaires ou branchés (ABS), les paraffine sulfonates, les alpha-oléfine sulfonates, les alcools gras éthoxylés, etc.

Au niveau des procédés, les principales matières premières « fossiles » utilisées sont les paraffines ou hydrocarbures, l'éthylène, le propylène et le benzène [4]. Après transformations (figure 2), ces dérivés conduisent à des intermédiaires comme des oléfines, des alcools gras ou des alkylbenzènes sur lesquels sont greffées, dans un second temps, les parties hydrophiles. Cette dernière étape met le plus souvent en œuvre des réactions de sulfonation, de sulfatation ou d'alcoxylation.

Après les années 70 , les deux critères cités précédemment sont restés valables mais, à ceux-ci se sont ajoutés d'autres critères liés principalement au devenir du tensioactif dans l'environnement après usage (toxicité, écotoxicité, biodégradabilité). Depuis, cette prise de conscience $n^{\prime}$ a fait que s'accroître et est renforcée, de nos jours, avec notamment les concepts de chimie verte et de développement durable ainsi que la future réglementation européenne Reach.

- Dans ce contexte, la valorisation de substrats lipophiles d'origine naturelle a été privilégiée et I'oléochimie a donc pris toute son importance dans la conception de tensioactifs respectueux de l'environnement.

La première génération de substrats lipophiles naturels correspond aux acides gras 1 ou aux esters méthyliques 2 issus respectivement de la saponification ou de la méthanolyse d'huiles ou de graisses triturées. À ce niveau, il est important de souligner que ces deux voies produisent parallèlement du glycérol pouvant ensuite constituer une source d'hydrophilie lors de la synthèse d'un tensioactif (éthers ou esters d'oligo- ou polyglycérol [5]).

Selon la composition intrinsèque des triglycérides de départ, ces acides et esters se caractériseront par des chaînes linéaires hydrocarbonées présentant un nombre pair de carbones compris entre 8 et 22 ainsi que par la présence ou non d'insaturation(s) et/ou d'une fonction hydroxyle (tableau 1).

Au-delà de la fabrication de savons qui est connue depuis plusieurs siècles, d'autres transformations chimiques des acides 1 et des esters L'oléochimie constitue ainsi une filière de choix pour accéder à une large gamme de substrats lipophiles naturels bien adaptés à la conception de nouveaux tensioactifs mixtes ou biotensioactifs.

\section{Sources de matières premières lipochimiques}

Avant les années 70 , les deux principaux critères à atteindre pour développer un tensioactif étaient : i) une rentabilité industrielle et économique et ii) des performances lors de son utilisation. Cette approche a abouti, après plusieurs décennies, à la commercialisation de tensioac-

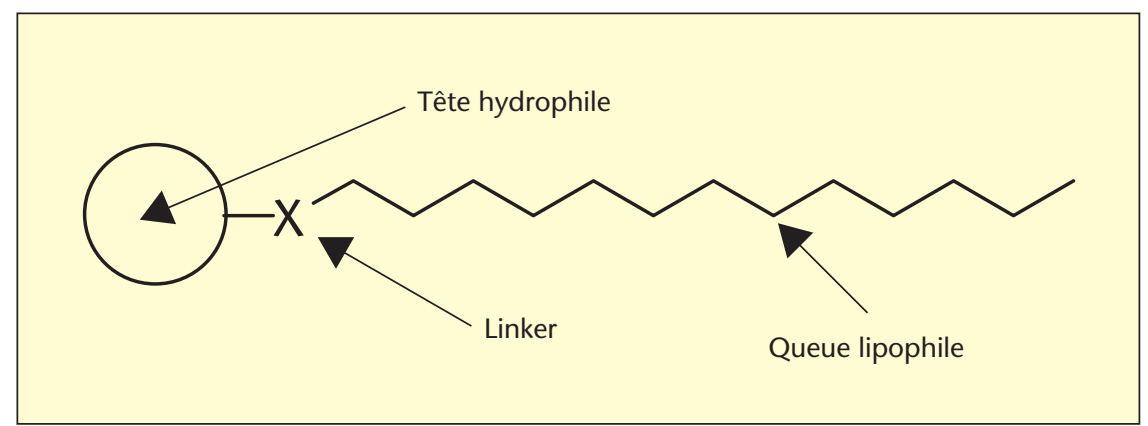

Figure 1. Structure simplifiée d'un dérivé tensioactif. 


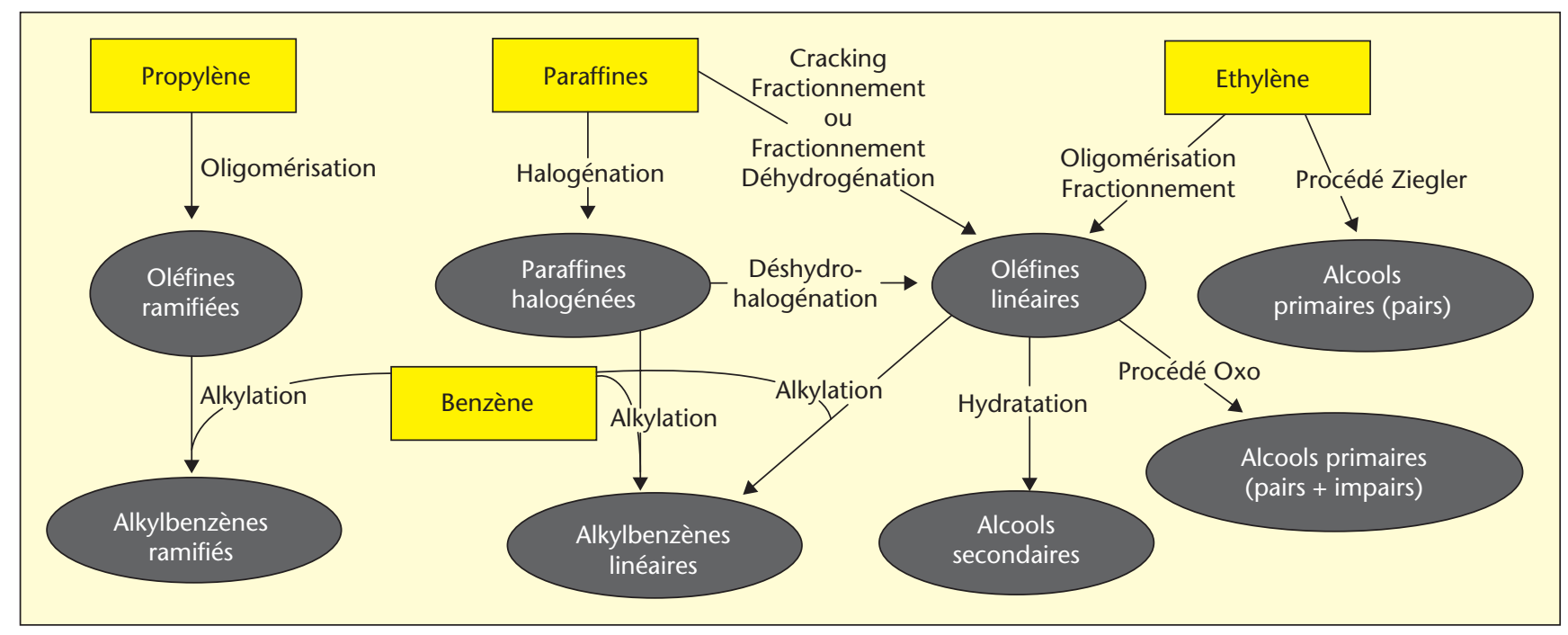

Figure 2. Accès à la partie lipophile de tensioactifs « non renouvelables».

Tableau 1. Nature des chaînes acyles des huiles et graisses les plus utilisées.

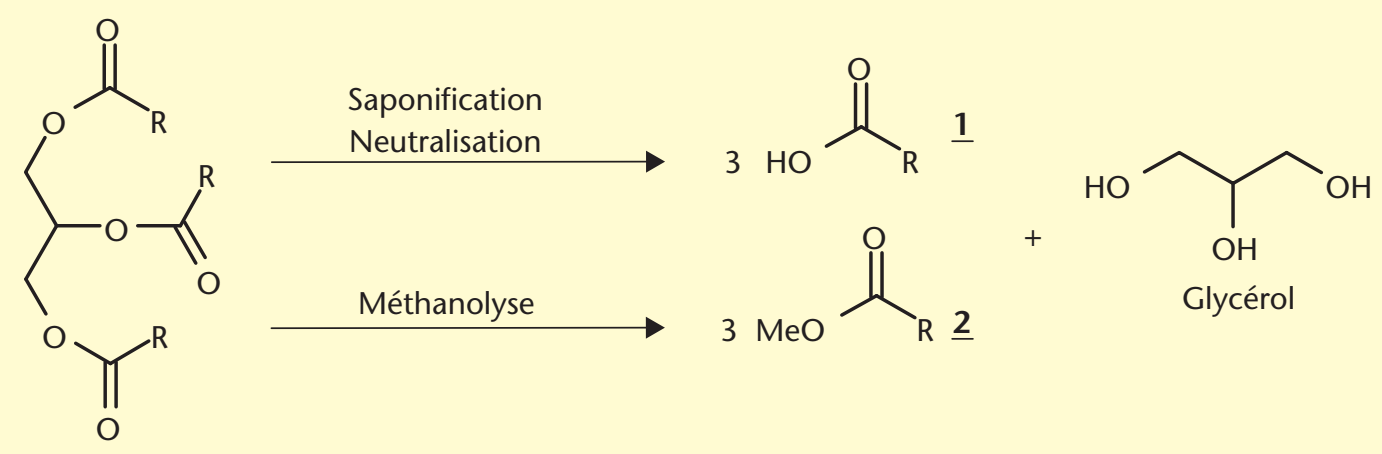

\begin{tabular}{|lcccccccc|}
\hline Chaînes R + 1 & Palmistes & Coprah & Palme & $\begin{array}{c}\text { Colza « low } \\
\text { erucic » }\end{array}$ & $\begin{array}{c}\text { Tournesol } \\
\text { classique }\end{array}$ & Soja & Ricin & Suif \\
\hline Caprylique C 8:0 & $3-10$ & $6-9$ & $/$ & $/$ & $/$ & $/$ & $/$ & $/$ \\
Caprique C 10:0 & $3-14$ & $6-10$ & $/$ & $/$ & $/$ & $/$ & $/$ & $/$ \\
Laurique C 12:0 & $37-52$ & $44-51$ & $/$ & $/$ & $/$ & $/$ & $/$ & $2-6$ \\
Myristique C 14:0 & $7-17$ & $13-18$ & $0-5$ & $0-1$ & $/$ & $/$ & $1-2$ & $20-33$ \\
Palmitique C 16:0 & $2-9$ & $8-10$ & $32-47$ & $1-5$ & $3-10$ & $7-10$ & $1-2$ \\
Stéarique C 18:2 & $1-3$ & $1-3$ & $2-8$ & $0-2$ & $1-10$ & $3-6$ & $1-2$ \\
Oléique C 18:1 & $11-23$ & $5-8$ & $40-52$ & $50-65$ & $15-40$ & $20-35$ & $4-9$ & $35-50$ \\
Ricinoléique C 18:1-OH & $/$ & $/$ & $/$ & $/$ & $/$ & $/$ & $86-92$ & $/$ \\
Linoléique C 18:2 & $1-3$ & $0-3$ & $5-11$ & $15-30$ & $45-70$ & $40-57$ & $3-6$ & $2-5$ \\
Linolénique C 18:3 & $/$ & $/$ & $/$ & $6-13$ & $0-1$ & $5-14$ & $/$ & $<1$ \\
Eicosénique C 20:1 & $/$ & $/$ & $/$ & $1-3$ & $<0,5$ & $<0,4$ & $/$ & $/$ \\
Béhénique C 22:0 & $/$ & $/$ & $/$ & $0-1$ & $<1$ & $<0,5$ & $/$ & $/$ \\
Euricique C 22:1 & $/$ & $/$ & $/$ & $0-5$ & $/$ & $/$ & $/$ \\
\hline
\end{tabular}

2 permettent, dans un deuxième temps, d'isoler des chaînes hydrocarbonées possédant les mêmes caractéristiques que celles des acides ou des esters de départ et se différenciant uniquement par la nature de leur fonction chimique terminale (figure 3).
La réduction d'un acide ou d'un ester conduira aux alcools gras 3 correspondants. Le traitement à l'ammoniac d'un alcool aboutira aux amines grasses 4 correspondantes, ellesmêmes précurseurs des alkyl isocyanates 5 . Enfin, des halogénures d'acide 6 seront issus de la réaction d'un acide gras avec un agent activant adapté.

Parallèlement à des développements industriels dans d'autres secteurs, la transformation d'acides gras insaturés tels que les acides oléique ou ricinoléique [6] (figure 4) constitue une 


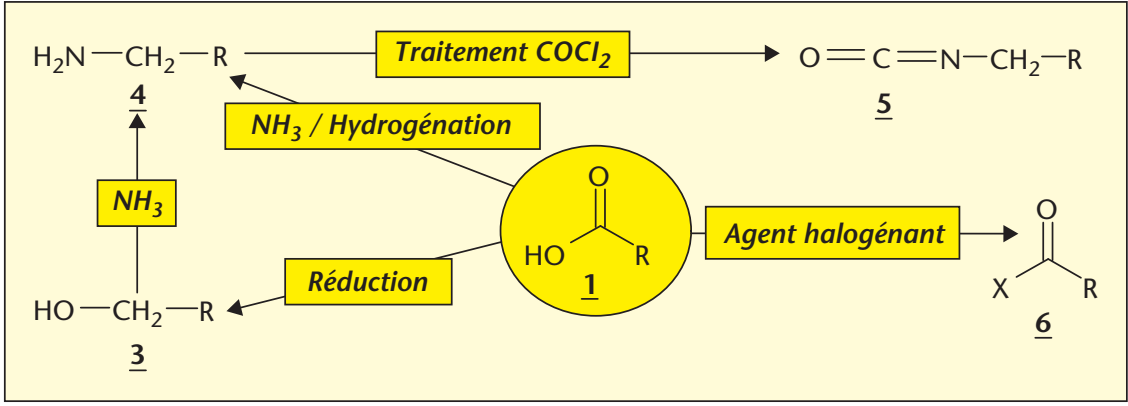

Figure 3. Exemples de transformations chimiques des acides 1.

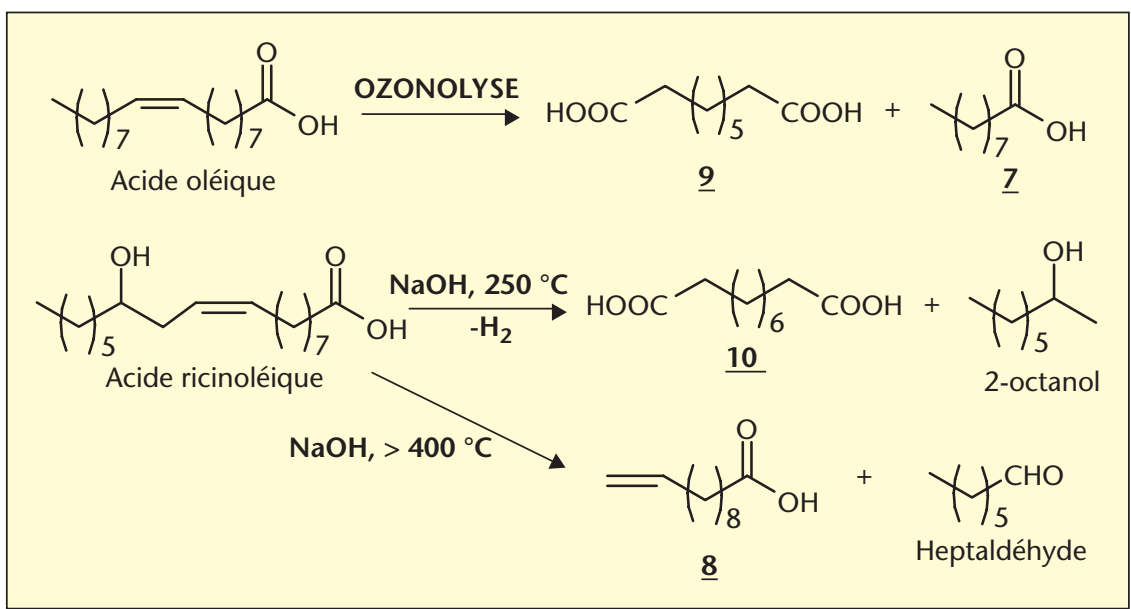

Figure 4. Modifications chimiques des acides gras insaturés.

voie intéressante pour synthétiser des dérivés possédant un nombre impair de carbones (les acides perlargonique 7 ou undécénoïque 8) ou une double fonctionnalité (les acides azélaïque 9 ou sébacique 10).

Finalement, des réactions de dimérisation peuvent également être envisagées pour obtenir de nouveaux substrats lipophiles se caractérisant par un nombre de carbones supérieur à 22 et/ou par la présence de ramifications (figure 5). À partir d'alcools gras linéaires purs ou en mélange, cette stratégie aboutira à des alcools alpha-ramifiés 11 , encore appelés alcools de Guerbet $[7,24]$. La dimérisation d'acides gras insaturés issus du « tall oil » par exemple permettra de former des acides dimères 12 acycliques, monocycliques ou bicycliques et possédant 36 atomes de carbone [8].

Ce rapide tour d'horizon non exhaustif de matières premières lipophiles issues de l'oléochimie montre le choix considérable qui s'offre à l'homme de l'art pour élaborer des structures tensioactives. Cette filière permet d'accéder à de nouvelles familles de molécules (biotensioactifs) et, par suite, de substituer progressi- vement des agents de surface d'origine totalement pétrochimique.

Au-delà des gains environnementaux, le secteur des oléagineux constitue donc une source d'innovation importante. La synergie tensioactifs/oléochimie constatée durant ces trois dernières décennies a probablement de beaux jours devant elle et ne pourra être qu'amplifiée compte tenu des axes actuellement pris dans le domaine des biocarburants (investissements industriels, valorisation des produits secondaires, etc.).

\section{Valorisation de l'oléochimie dans le domaine des tensioactifs}

Comme évoqué en introduction, le caractère amphiphile d'un tensioactif apporte à cette structure des propriétés spécifiques aux interfaces. Ces propriétés se traduisent concrètement par différents phénomènes qui peuvent être valorisés dans diverses applications.

La présence de tensioactifs dans une solution aqueuse va induire un abaissement de la tension interfaciale eau/air qui va, ensuite, favoriser l'étalement de la solution sur une surface donnée. Son pouvoir mouillant est ainsi amélioré.

À partir d'une certaine concentration appelée concentration micellaire critique (CMC), les tensioactifs en solution aqueuse vont s'autoorganiser en micelles de façon à minimiser les interactions hydrophobes. Dans cette configuration, des matières organiques peu voire pas solubles dans l'eau pourront s'incorporer dans le cœur lipophile des micelles (figure 6) et une solubilisation sera alors observée.

Les propriétés mouillantes et solubilisantes d'un tensioactif sont généralement recherchées simultanément et mises à profit en détergence ménagère ou industrielle.

Les tensioactifs participent également au phénomène de dispersion de particules liquides dans une autre phase liquide non miscible, encore appelé émulsification (figure 7). En fonction du protocole d'émulsification ainsi que des propriétés physico-chimiques du système tensioactif utilisé, on aura soit des émulsions huile dans eau $(H / E)$, soit des émulsions eau dans

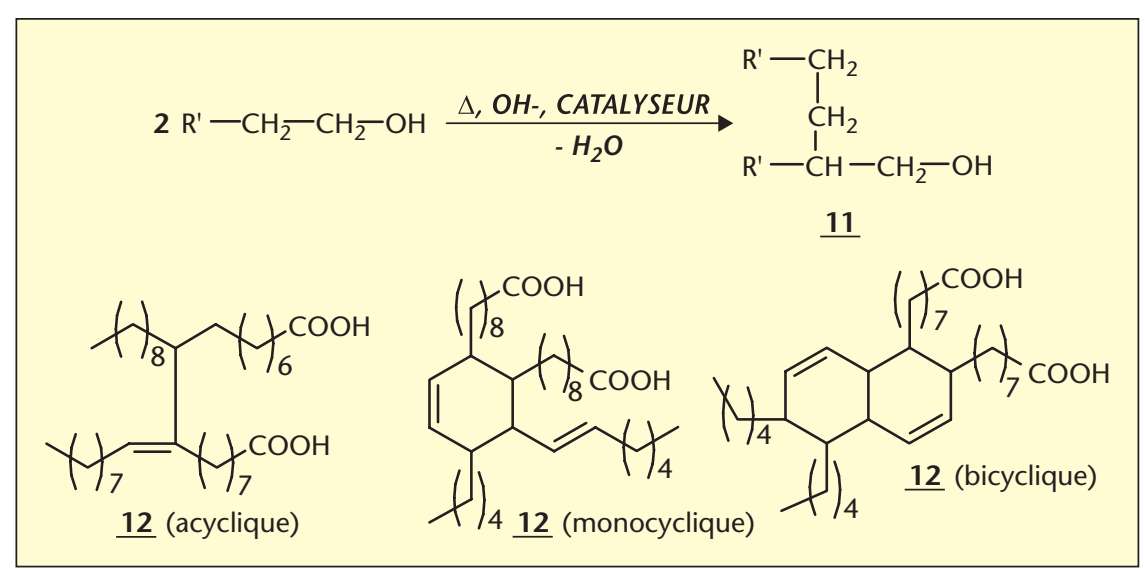

Figure 5. Réactions de dimérisation d'alcools gras ou d'acides gras insaturés. 


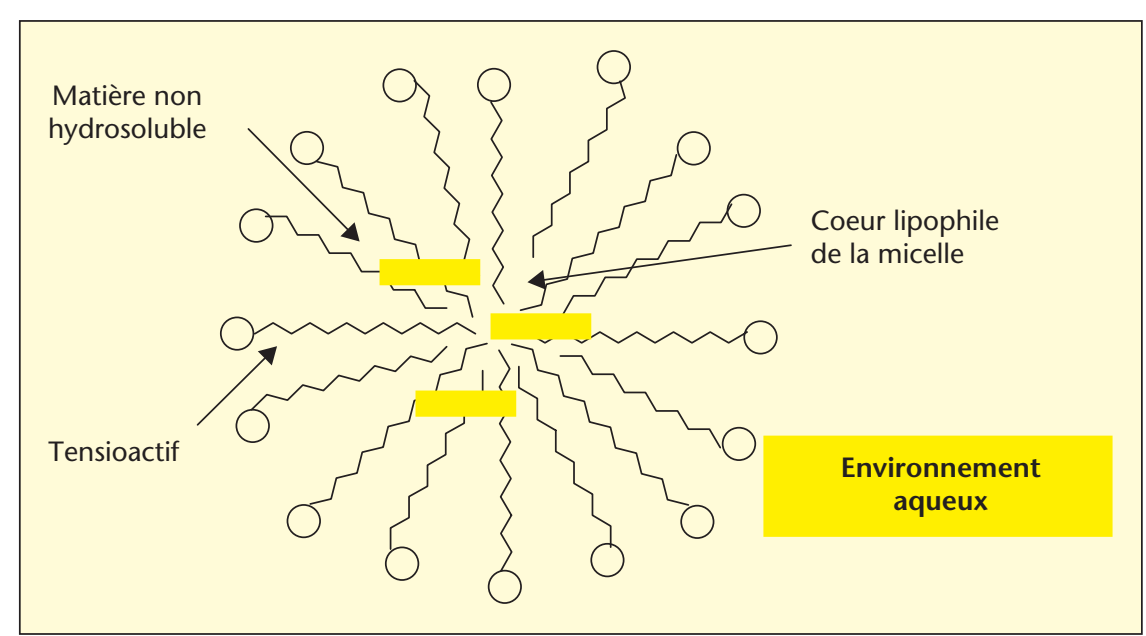

Figure 6. Solubilisation d'un substrat lipophile dans une micelle.

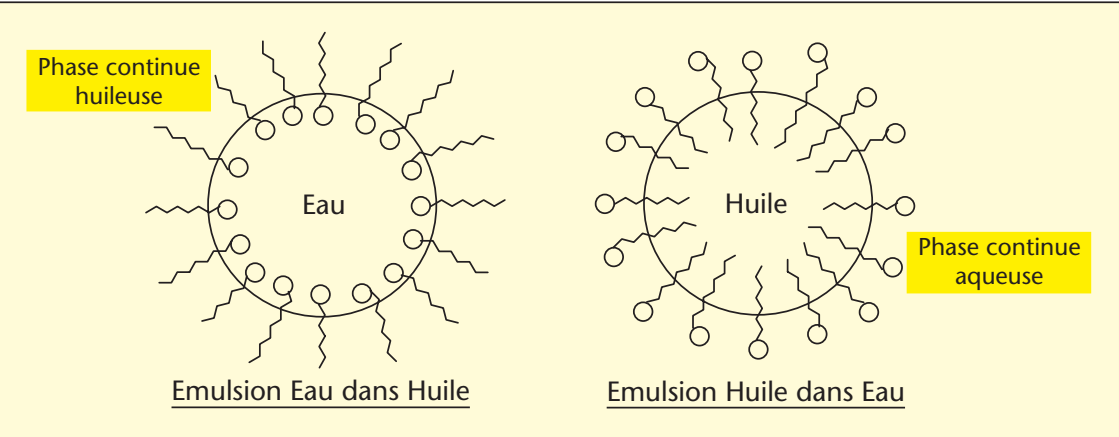

Figure 7. Structures microscopiques d'émulsions E/H et $H / E$.

huile $(E / H)$. Cette aptitude à émulsifier est largement valorisée pour formuler des produits cosmétiques, pharmaceutiques, vétérinaires ou phytosanitaires.

Finalement, les agents de surface sont capables, en présence d'eau et dans certaines conditions, de former des phases cristallines liquides ainsi que des vésicules ou liposomes. Les vésicules sont des objets supramoléculaires sphériques constitués d'un cœur aqueux et d'une ou plusieurs membrane(s) de tensioactifs (figure 8). Ces objets constituent des réservoirs potentiels à l'intérieur desquels pourront être encapsulés des substrats hydrophiles ou lipophiles tels que des actifs médicamenteux, des parfums, du matériel génétique (transfection $\left.d^{\prime} A D N\right)$, etc.

Toutes ces propriétés sont directement régies par la structure intrinsèque du tensioactif : géométrie générale, poids moléculaire, nature des liaisons chimiques $X$ reliant la partie polaire à la queue lipophile, présence de groupements ioniques, balance hydrophile lipophile (HLB). Selon la ou les fonctionnalité(s) des substrats de départ ainsi que les stœchiométries engagées, il sera possible d'accéder à des structures de type mono-, bi-, tricaténaire, etc., gemini

Figure 8. Coupe transversale d'un liposome.
La polarité relative des substrats de départ est également primordiale car elle oriente la HLB du tensioactif, elle-même corrélée aux propriétés finales du tensioactif (figure 9).

De manière similaire aux matières premières pétrochimiques, l'oléochimie permet de moduler tous ces paramètres. Elle ouvre même de nouvelles perspectives avec notamment l'utilisation d'acide 12-hydroxystéarique (dérivé de l'acide ricinoléique) pour préparer des tensioactifs polymères [11]. En effet, la présence simultanée d'une fonction acide carboxylique et hydroxyle permet d'envisager, avant ou au cours du greffage de la tête polaire, des réactions de polyestérification (figure 10). Cette famille de composés présente, en général, des performances accrues de stabilisation d'émulsions compte tenu de leurs masses moléculaires relativement élevées par rapport à un agent de surface classique (entre 3000 et $8000 \mathrm{~g} / \mathrm{mol}$ ).

Au-delà des aspects structuraux et applicatifs, l'intérêt d'utiliser des matières premières oléochimiques réside dans le caractère renouvelable de ces ressources végétales ou animales. L'épuisement à court ou moyen terme de nos ressources fossiles ainsi que la difficulté croissante à exploiter de nouveaux gisements ne font que renforcer cet argument.

Finalement, l'atout de l'oléochimie qui peutêtre se distingue le plus se situe au niveau des propriétés environnementales des biotensioactifs finaux. Leurs profils toxicologiques et écotoxicologiques sont, dans la plupart des cas, très satisfaisants et répondent bien aux exigences économiques et réglementaires actuelles. Ils se traduisent par des biodégradabilités rapide ou intrinsèque élevées (selon ligne directrice OCDE 301 A à F ou OCDE 302), de faibles toxicités vis-à-vis de l'homme et des différents niveaux trophiques de l'écosystème (daphnies-

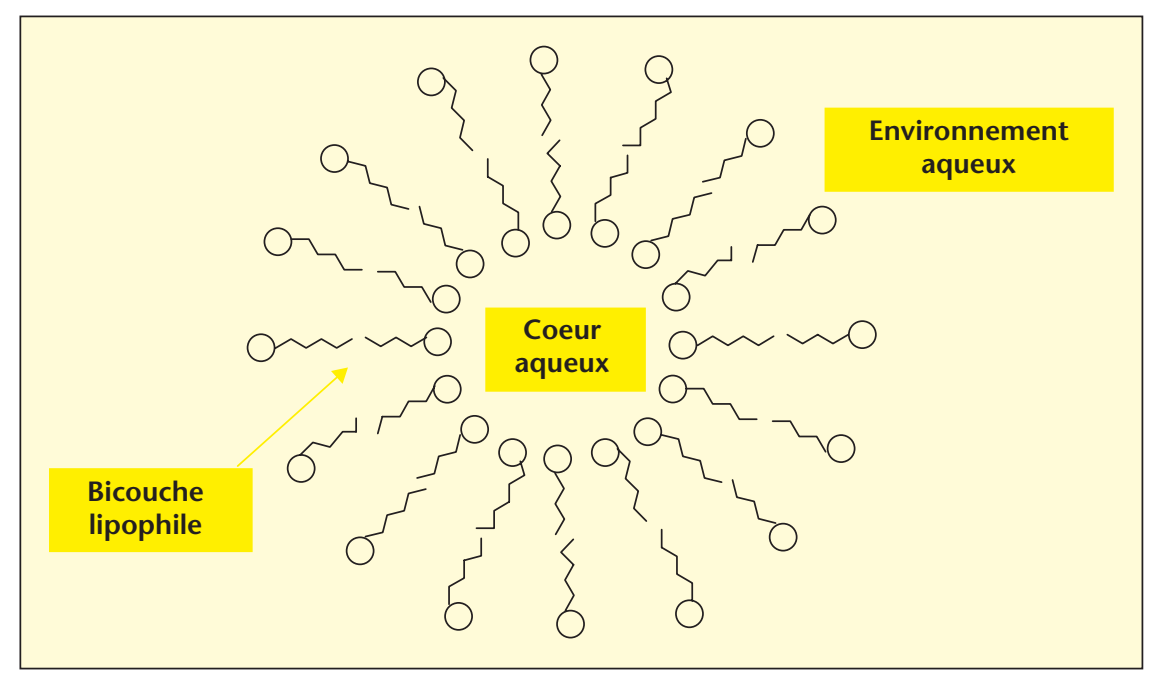




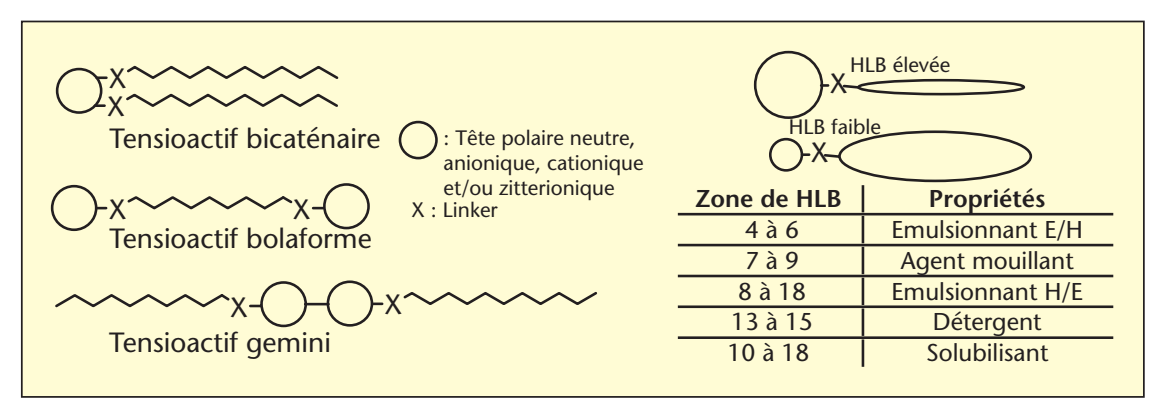

Figure 9. Géométrie variable d'un tensioactif - Corrélation HLB/propriétés.

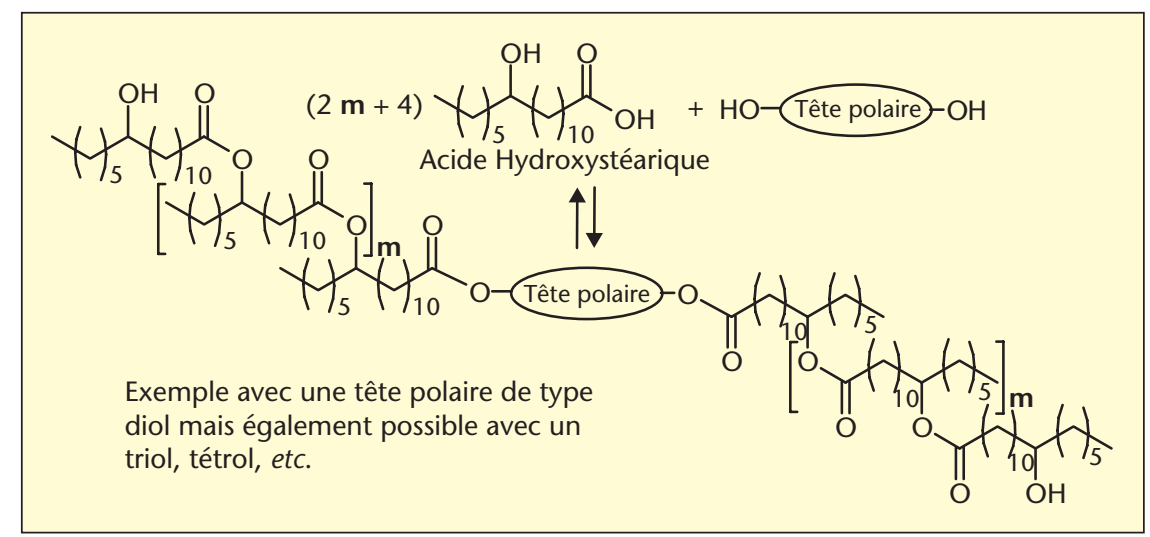

Figure 10. Synthèse de tensioactifs polymères de type PolyHydroxyStéarate.

selon OCDE 202, algues selon OCDE 201, poissons selon OCDE 203) et une bonne biocompatibilité avec des tolérances cutanée et oculaire accrues (tests HETCAM, RBCA, patch tests, sensibilisation Marzulli-Maibach).

\section{Quelques mots sur la partie hydrophile du tensioactif}

Avant d'aborder la description d'exemples de biotensioactifs, il semble important de rappeler préalablement les principales sources naturelles d'hydrophilie utilisées pour leurs synthèses.

\section{Sources d'hydrophilie à base de sucres ou de dérivés}

Les sucres (figure 11) constituent sans aucun doute une matière première polaire de choix pour l'élaboration de biotensioactifs. Leur point commun est de posséder un nombre important de fonctions hydroxyle participant à la formation de réseaux de liaisons hydrogène en présence d'eau. Selon leur famille (hexose ou pentose) et/ou leurs poids moléculaires (mono-, di-, tri-, oligo- ou polysaccharides), ils permettront de moduler aisément la HLB de l'agent de surface ainsi que sa géométrie générale.

À ce jour, les principales sources sont d'origine végétale : blé, maïs, pomme de terre, manioc, alpha-1,6. Après hydrolyse chimique et/ou enzymatique, ce polymère conduit au glucose 13 constitué d'une seule unité sucre. Une hydrolyse contrôlée est également envisageable et aboutira à des oligoglucoses 14 (maltodextrines) présentant un nombre variable de motifs glucose.

À partir de la betterave ou de la canne à sucre, est fabriqué, après extraction et cristallisation, le saccharose 15 correspondant à un dissacharide composé d'une unité glucose et d'une unité fructose. Ce disaccharide est largement utilisé dans l'industrie agroalimentaire pour son pouvoir sucrant.

L'industrie sylvicole est aussi une source notable de sucres avec notamment la cellulose et I'hémicellulose formant une structure fibreuse extracellulaire servant de squelette aux plantes (écorce de boulot, de hêtre, etc.).

La cellulose est un homopolymère linéaire de glucose présentant non plus des liaisons glucosidiques alpha-1,4 mais bêta-1,4. L'hémicellulose est un hétéropolymère plus complexe plus ou moins riche en résidus xylose 16 et arabinose 17 . Après extraction, hydrolyse et purification, ce polymère permettra $\mathrm{d}$ 'isoler ces deux monosaccharides faisant partie de la famille des pentoses.

Enfin, les racines de chicorée et les tubercules d'artichaut sont valorisés dans la fabrication de différents grades d'inuline. L'inuline est un polymère de fructose de la famille des fructanes se caractérisant par des liaisons glycosidiques bêta-2,1, des degrés de polymérisation compris entre 2 et 100 et la présence éventuelle d'un motif glucose terminal. L'hydrolyse totale ou partielle de ces grades d'inuline aboutira à des oligofructoses 18 ou au fructose pur 19. Finalement, des transformations chimiques, enzymatiques ou biotechnologiques des mono-, di-, ou oligosaccharides cités précé-

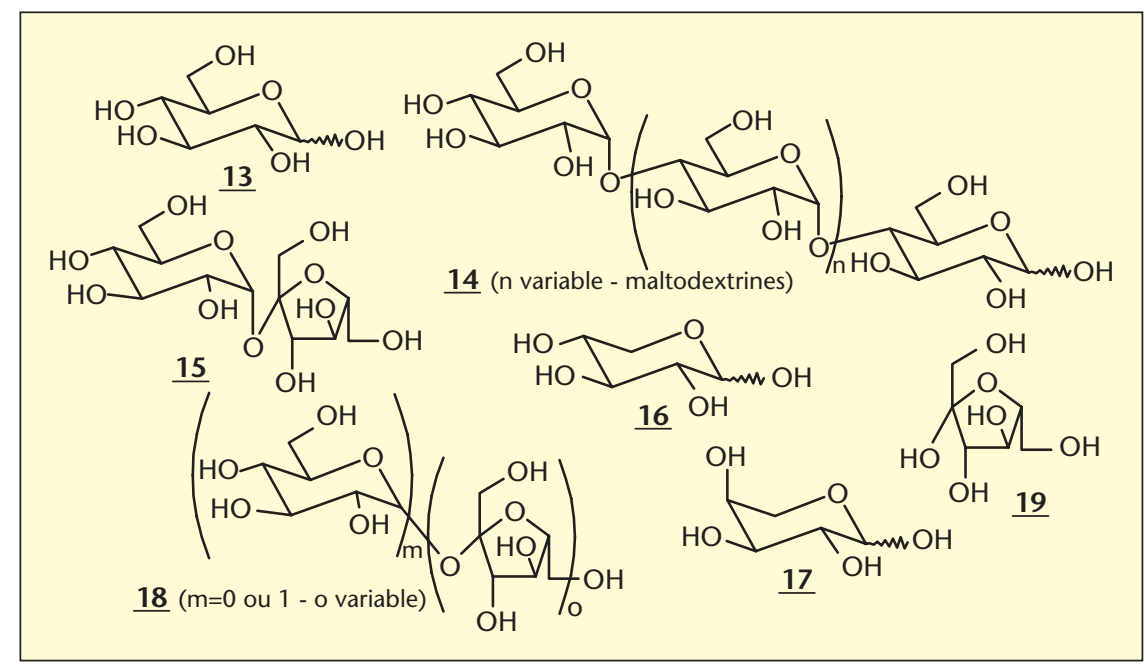

Figure 11. Exemples de sucres disponibles à l'échelle industrielle. 
demment peuvent conduire, dans un deuxième temps, à des polyols comme le sorbitol ou le xylitol ou à des structures saccharidiques macrocycliques telles que les cyclodextrines par exemple.

\section{Sources d'hydrophilie issues de dérivés protéiques}

Les acides aminés ou hydrolysats de protéines sont aussi de bons candidats pour jouer le rôle de tête polaire. Après «lipophilisation", ils conduisent respectivement à des lipoaminoacides ou à des lipoprotéines présentant un ou plusieurs motif(s) hydrophile(s) anionique(s) de type carboxylate.

Il existe vingt acides aminés naturels regroupés en fonction de la nature de leur chaîne latérale : non polaire neutre (phénylalanine, leucine, isoleucine, proline, valine, etc.), polaire basique (arginine et lysine) et polaire acide (acide glutamique et acide aspartique). Outre leur extraction de produits naturels, différentes méthodes de synthèses chimiques ou enzymatiques existent.

La composition des hydrolysats de protéines dépend de l'aminogramme des matières premières de départ ainsi que des conditions d'hydrolyse mises en œuvre. Les protéines végétales proviennent, le plus souvent, de « coproduits » de la transformation des plantes de grande culture: les glutens de blé ou de maïs issus de l'amidonnerie ou les concentrats protéiques ou tourteaux isolés après extraction de I'huile des oléagineux. Les protéines animales sont généralement des extraits (collagène ou gélatine, albumine, caséine, etc.). Elles sont plus chères que les protéines végétales. La tendance actuelle ne privilégie pas I'utilisation de cette source d'hydrophilie du moins pour des usages en cosmétique ou en alimentaire. Les protéines animales peuvent en effet souffrir d'une image négative liée notamment à la maladie de la « vache folle » (encéphalopathie spongiforme bovine - ESB).

Finalement, I'utilisation à l'échelle industrielle de peptides ou d'oligopeptides est très peu répandue. Le coût de fabrication de ce type de matières premières (synthèse peptidique multiétapes) reste encore onéreux pour la préparation de tensioactifs de spécialités (lipopeptides). On peut citer toutefois I'aspartame, correspondant à un dipeptide constitué d'un motif acide aspartique et d'un motif phénylalanine estérifié. Ce dipeptide est valorisé en agroalimentaire pour ses propriétés édulcorantes. Son prix pourrait éventuellement être adapté à des applications à haute valeur ajoutée mais le greffage de chaîne(s) lipophile(s) n'a, à notre connaissance, jamais été envisagé pour des raisons de stabilité.

\section{Exemples de biotensioactifs préparés à l'échelle industrielle}

Cette dernière partie a pour objectif de décrire succinctement les conditions opératoires [12] permettant de créer, dans des conditions efficaces et respectueuses de l'environnement, la liaison covalente reliant les parties hydrophile et lipophile du tensioactif.

\section{Biotensioactifs saccharidiques}

Les esters de polyol 20 sont des dérivés développés depuis plusieurs décennies et obtenus par estérification d'un polyol comme le sorbitol ou le mannitol (épimère en $\mathrm{C}$-2), en présence d'un acide gras 1 pur ou d'une coupe et d'un catalyseur adapté (figure 12).

Les réactions sont conduites sans solvant par dispersion du polyol dans l'acide gras fondu. Les sous-produits générés sont de l'eau et un décantat que I'on peut recycler en tant que pied de réaction. Sur la figure 12 , seul un monoester de sorbitan/mannitan a été représenté mais les milieux réactionnels sont relativement plus complexes. Des réactions de déshydratation intramoléculaire se produisent préalablement à l'estérification pour conduire à un mélange de sorbitol, de sorbitan et d'isosorbide. Selon la stœchiométrie en acide gras mise en jeu ainsi que la réactivité des différentes fonctions alcools, on formera des mono-, di-, tri- voire tétraesters.

Ces esters de polyols constituent des émulsionnants lipophiles possédant une HLB comprise entre 2 et 8 . Ils sont utilisés en cosmétique (gamme Montane ${ }^{\circledR}$ par exemple) ou en santé animale (gamme Montanide ${ }^{\circledR}$ - vaccination des bovins et des porcs contre la fièvre aphteuse). Leurs biodégradabilité et écotoxicité sont satisfaisantes [13]. Dans la majorité des cas, ils sont couplés avec leurs homologues éthoxylés («Polysorbates» - gamme Montanox ${ }^{\circledR}$ par exemple) de façon à accroître la fenêtre de HLB et à optimiser les stabilités des émulsions.

À ce jour, les meilleurs exemples de produits issus de la glycochimie sont les AlkylPolyGlucosides 21 (APG) obtenus à partir de glucose 13 cristallisé et d'un alcool gras 3 pur ou d'une coupe (figure 13).

Les réactions de glycosylation sont conduites selon les conditions de Fischer [14] qui consistent à activer la position anomérique du sucre par un catalyseur acide et à éliminer l'eau au fur et à mesure de sa formation. Là encore, les réactions sont conduites sans solvant avec le glucose en suspension dans un excès d'alcool gras fondu. Les sous-produits générés sont de l'eau et un résidu de sucre. Le greffage de l'alcool sur le sucre activé s'accompagne très souvent d'une polymérisation de la tête polaire saccharidique. Les degrés de polymérisation (DP), compris généralement entre 1,3 et 1,6 [15], sont directement liés aux conditions expérimentales et peuvent moduler sensiblement la HLB de I'APG final. Les réactions n'étant pas stéréocontrôlées, on sous-entend lorsque l'on dit APG un mélange de stéréoisomères alpha, bêta, furano-, pyrano-, que l'on ne purifie pas pour des raisons économiques.

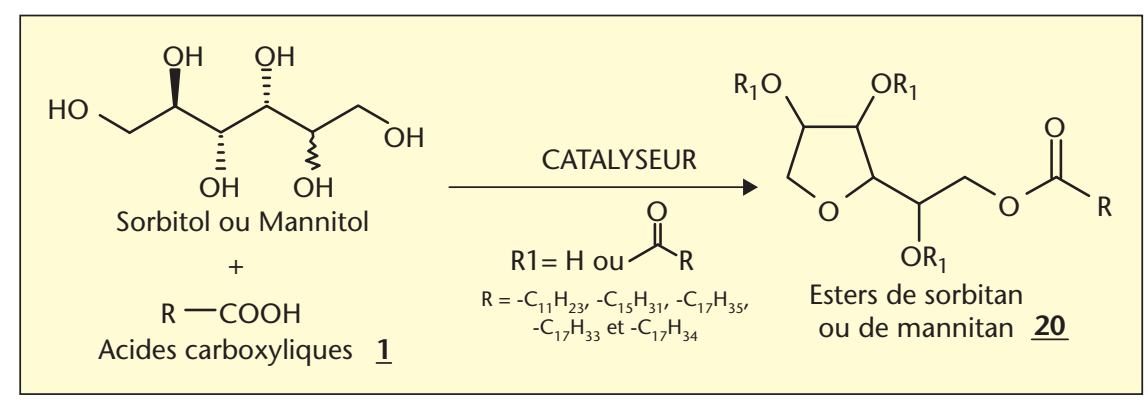

Figure 12. Préparation d'esters de sorbitan ou de mannitan.

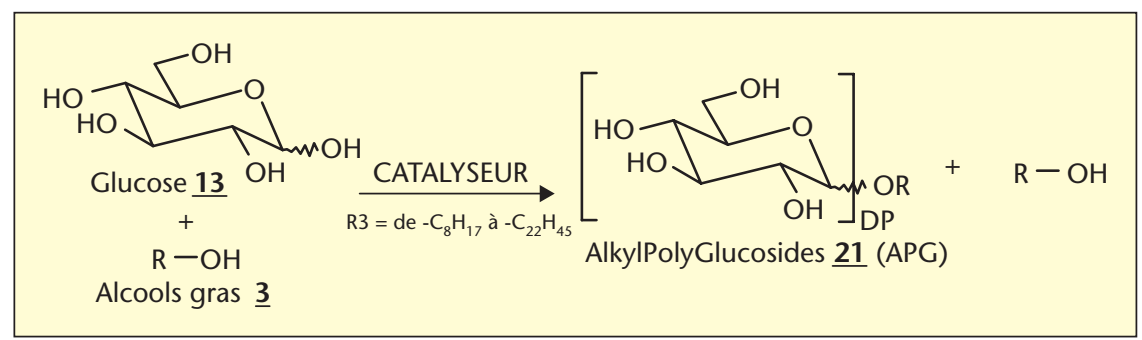

Figure 13. Glucosylation d'un alcool gras selon les conditions de Fischer. 
En fin de process, l'excès d'alcool est soit laissé dans le milieu soit évaporé. Pour des chaînes longues $(R \geq 14)$, I'APG présente des propriétés émulsionnantes huile dans eau (gamme Montanov ${ }^{\circledR}$ par exemple). En revanche pour des chaînes plus courtes $(R<14)$, l'APG est commercialisé en solution aqueuse et aura des propriétés solubilisantes, mouillantes ou moussantes. Ces APG sont principalement introduits dans des formules cosmétiques (crèmes, gels douche, etc.) ou détergentes (produit vaisselle). Ils sont biodégradables et non écotoxiques.

La glycosylation d'alcools de Guerbet 11 est également envisageable [16]. On peut citer notamment la préparation d'octyldodécyl xyloside 22 (gamme Fluidanov ${ }^{\circledR}$ par exemple figure 14) valorisé en cosmétique en tant que co-émulsionnant eau dans huile. Son originalité repose, d'une part, sur la présence d'une tête xylosidique (alkylpolyxyloside - APX), et, d'autre part, sur son aspect liquide permettant des procédés d'émulsification à froid.

Dans une seconde phase, le coût de fabrication des APG permet de les utiliser comme matières premières dans de nouvelles fonctionnalisations. Les principaux exemples commerciaux de dérivés d'APG sont les esters de méthylglucosides 23 [17] (gammes Glucam ${ }^{\circledR}$, Glucamate ${ }^{\circledR}$, Clucate ${ }^{\circledR}$ ou Grillocose ${ }^{\circledR}$ - figure 14) et des APG anioniques (hémisulfosuccinates, citrates, tartrates) ou cationiques (gamme Sugarquat ${ }^{\circledR}$ ).

Les glucamides 24 sont synthétisés en deux étapes (figure 15) : i) réaction du glucose 13 avec la méthylamine et ii) amidification de la $\mathrm{N}$-méthylglucamine intermédiaire en présence d'un ester méthylique 2 .

Contrairement aux APG, ces composés présentent qu'un seul motif glucose et sont donc moins hydrosolubles. Une acétylation optionnelle de la $\mathrm{N}$-méthylglucamine résiduelle permet finalement d'éviter toutes traces de nitrosamines dans les produits finaux. Ils sont majoritairement utilisés en détergence avec des propriétés applicatives et environnementales proches de celles des APG.

Les esters de saccharose constituent également une classe intéressante de biotensioactifs saccharidiques [18]. La principale difficulté, compte tenu du nombre important de fonctions hydroxyles du sucre de départ, réside dans l'obtention de dérivés principalement monosubstitués. Différentes méthodes existent pour atteindre une régiosélectivité : catalyses enzymatiques, acylation par des chlorures d'acide, purification par cristallisation ou par extraction, etc. Toutefois, la voie la mieux adaptée à l'échelle industrielle reste, à ce jour, celle de la transestérification suivie d'une purification des monoesters. Ces composés sont utilisés en tant qu'émulsionnants dans I'ali-

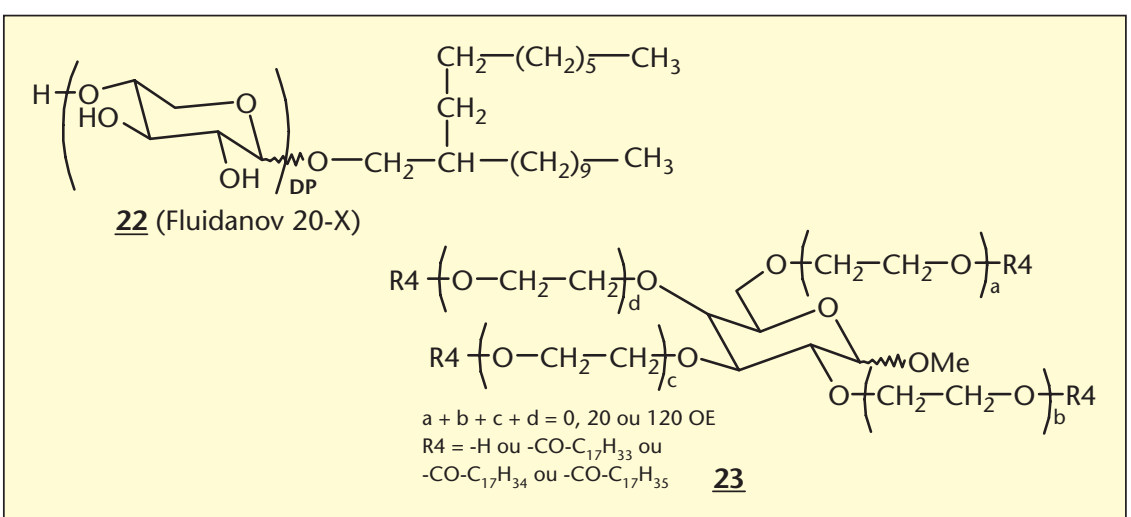

Figure 14. Structures chimiques d'un APX 22 et de dérivés d'APG 23.

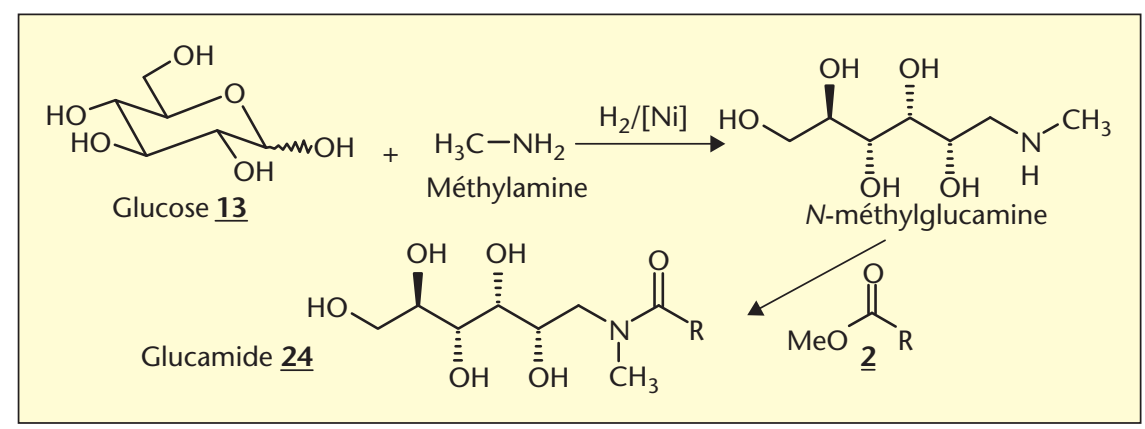

Figure 15. Synthèse en deux étapes des glucamides 24. mentaire ou la cosmétique (gammes Crodesta ${ }^{\circledR}$, Sefose $^{\circledR}$, etc.). D'autres fonctionnalisations, comme par exemple la préparation d'alkyl carbamates de saccharose [19], peuvent être évoquées, mais celles-ci ne connaissent pas, pour le moment, de développement industriel.

Finalement, l'obtention de tensioactifs glycosidiques possédant une hydrophilie accrue est possible en fonctionnalisant des oligosaccharides (DP > 2) $[20,26]$. Leur HLB dépendera alors du DP de l'oligosaccharide de départ ainsi que du nombre de chaînes lipophiles greffées (Degré de Substitution - DS). Un exemple commercialisé récemment en cosmétique correspond aux alkyl carbamates d'oligofructoses (gamme Inutec ${ }^{\circledR}$ ) obtenus à partir d'alkyl isocyanates 5 et d'hydrolysats d'inuline 18 [21].

\section{Les lipoaminoacides}

Les lipoaminoacides 25 et plus généralement les lipoprotéines sont obtenus selon les conditions de Schotten-Baumann $[22,27]$ par acylation d'un ou plusieurs résidu(s) acide alphaaminé par un chlorure d'acide 6 en présence d'une base (figure 16).

Ces réactions sont conduites en milieu aqueux ou hydro-organique (eau/alcool, eau/cétone) selon la solubilité initiale du dérivé protéique de départ.

Dans le cas d'un acide aminé présentant une fonction réactive sur sa chaîne latérale (amine, hydroxyle ou guanidine), les conditions ci-dessus ne permettent pas une acylation régiosélective et les milieux réactionnels se

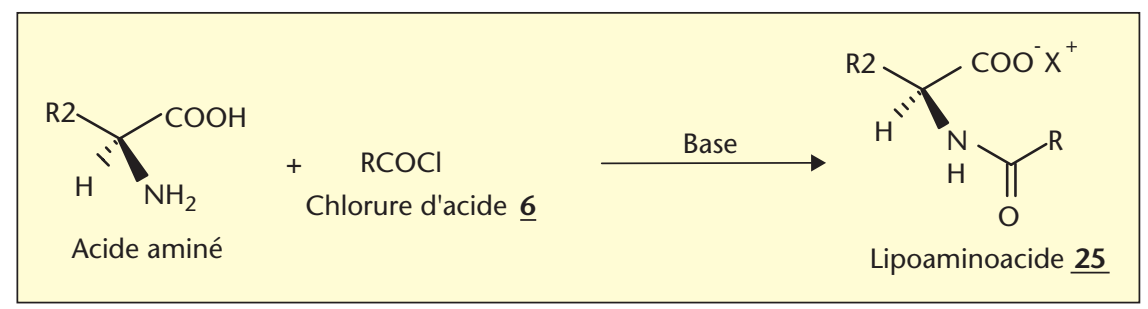

Figure 16. Acylation d'un acide aminé par un chlorure d'acide 6. 
caractériseront par des mélanges de dérivés mono- et diacylés.

Contrairement aux hydrolysats de protéines animales ou végétales, l'utilisation d'acide(s) aminé(s) limitent les problèmes de reproductibilité en termes d'odeur, de couleur et de performances applicatives.

En fin d'acylation, deux alternatives sont possibles. La première consiste à régler le $\mathrm{pH} d u$ milieu final autour de 7 et les lipoaminoacides (gamme Protéol ${ }^{\circledR}$ par exemple) sont alors commercialisés tel quel en solution aqueuse. Les propriétés moussantes de ces tensioactifs anioniques sont valorisées en cosmétique pour formuler des gels douche, des lotions nettoyantes ou des shampoings. La seconde alternative consiste à faire précipiter le lipoaminoacide en acidifiant le mélange réactionnel à $\mathrm{pH} \sim 2$ puis à réaliser plusieurs opérations de lavage. Cette procédure permet d'éliminer tous les sels générés au cours de l'acylation et par conséquent $d^{\prime}$ 'isoler un lipoaminoacide sous la forme d'une poudre présentant une pureté élevée.

Compte tenu du surcoût engendré par les étapes de filtration/séchage, cette voie est privilégiée pour la mise au point d'actifs cosmétiques (tableau 2) comme des anti-âges (raffermissant, apaisant), des agents dépigmentants ou amincissants par exemple.

Selon la même stratégie, des dichlorures d'acide peuvent être engagés dans des réactions d'acylation d'acides aminés (figure 17). Leur double fonctionnalité permet d'envisager la formation de lipoaminoacides bolaformes. À titre d'exemple, la faible hydrosolubilité de l'acide azélaïque formulé en cosmétique pour ses propriétés bactériostatique, bactéricide, sébo-régulatrice et éclaircissante a récemment été améliorée $[23,28]$ en greffant un motif glycinate de potassium à chacune de ses extrémités (Azeloglicina ${ }^{\circledR}$ ).

\section{Conclusion}

L'oléochimie joue un rôle primordial dans le domaine des tensioactifs et, plus particulièrement, dans le domaine des biotensioactifs. Elle constitue, à ce jour, la seule voie pour fabriquer des agents de surface totalement d'origine naturelle.

Le large éventail de chaînes hydrocarbonées qu'elle offre est bien adapté à la « lipophilisation » de polyols, de sucres ou de dérivés protéiques. Ce choix permet de moduler différents paramètres comme la HLB ou la nature du linker $X$ et, par suite, les propriétés physicochimiques et applicatives des tensioactifs finaux. Dans ce contexte, l'oléochimie constitue une source d'innovation importante pour développer de nouveaux tensioactifs performants et respectueux de l'environnement.

Contrairement aux ressources pétrochimiques qui suscitent actuellement beaucoup d'interrogations, la disponibilité des ressources oléochimiques ne pose pas de problème particulier et est renforcée compte tenu des nombreux axes de développement menés parallèlement dans d'autres secteurs.

En conclusion, la contribution mutuelle déjà constatée entre le secteur des oléagineux et celui des tensioactifs a mis en évidence de nombreux points forts et va très probablement se poursuivre dans l'avenir.

Tableau 2. Exemples d'actifs cosmétiques à base de lipoaminoacides.

\begin{tabular}{|c|c|c|c|c|}
\hline Acide aminé - R2 & Chaîne acyle-CO-R & $\begin{array}{l}\text { Contre- } \\
\text { ion } \mathrm{X}\end{array}$ & Propriété & Nom commercial \\
\hline Glycine & Octanoyle & $\mathrm{H}$ & Sébo-régulateur & Lipacide C-8G \\
\hline Glycine & Undécénoyle & $\mathrm{H}$ & $\begin{array}{c}\text { Sébo-régulateur Anti- } \\
\text { pelliculaire }\end{array}$ & Lipacide UG \\
\hline Phénylalanine & Undécénoyle & $\mathrm{H}$ & Dépigmentant & Sepiwhite MSH \\
\hline Hydroxyproline & Palmitoyle & $\mathrm{H}$ & Raffermissant & Sepilift DPHP \\
\hline Proline & Lauroyle & $\mathrm{H}$ & Amincissant & Adiposlim \\
\hline Proline & Palmitoyle & H & Apaisant & Sepicalm VG \\
\hline
\end{tabular}

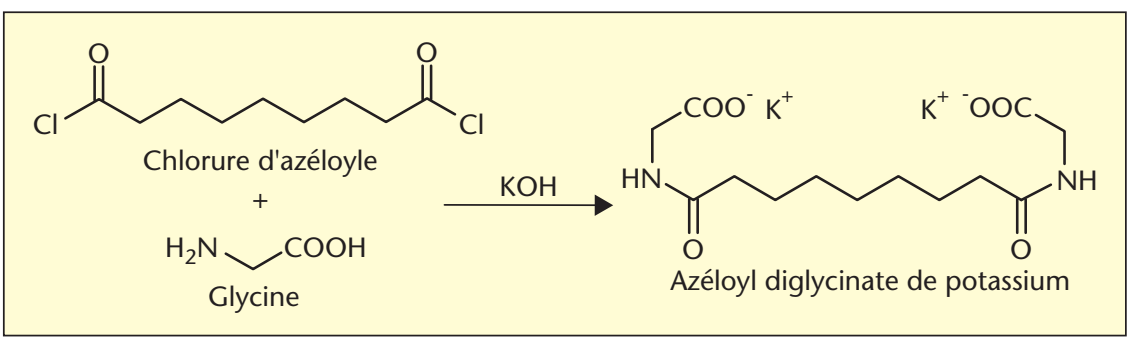

Figure 17. Acylation de la glycine par le chlorure d'azéloyle.

\section{RÉFÉRENCES}

1. MONTAGNE X. In : Biocarburants - les carburants liquides. La chimie verte. Paris : Lavoisier, 2006 : 419-45.

2. MOULOUNGUI $Z$, PELET $S$, EYCHENNE $V$, MATEO S. In : Lipochimie. La chimie verte. Paris : Lavoisier, 2006 : 305-52.

3. AGRICE/ADEME. Tensioactifs et oléagineux Etude sur les matières premières oléagineuses disponibles sur le marché européen. Novembre 2001.

4. HO TAN TAI L. Principaux tensioactifs et leurs procédés de synthèse. Détergents et produits de soins corporels. In : Paris : Dunod, 1999 : 15-32.

5. ANSMANN A, KAWA R, STRAUSS G. VON KRIES R. Polyol polyhydroxystearates, 1995, WO 9534528.

6. SWERN D. Bailey's industrial oil and fat products. vol. 1 (4). Wiley Interscience, 1979.

7. O'LENICK JA. « Guerbet chemistry ». Journal of Surfactants and Detergents 2001; 4(3); (2001).

8. OTHMER K. Dimer acids. Encyclopedia of chemical technology. Wiley Interscience, 1979 : 76881 ; [vol. 7 (3)].

9. INFANTE MR, PEREZ L, PINAZO A, et al. Amino acid-based surfactants. CR Chimie 2005; 7 : 583-92.

10. GUILBOT J. Synthèse, évaluation et développement de nouveaux amphiphiles bolaformes pour la cosmétique, la santé et l'agrochimie. Thèse de doctorat de l'Université de Rennes 11999.

11. BAKER AS. Block or graft copolymers and their use as surfactants. US 4203877, 1980.

12. BEHLER A, BIERMANN M, HILL K, RATHS HC, SAINT VICTOR ME, UPHUES G. In : Industrial surfactant syntheses. Surfactant science series. Marcel Dekker, 2001 : 1-44.

13. EPA. Test plan for the sorbitan esters category of the aliphatic esters chemicals. 2003. www.epa.gov/oppt/chemrtk/alipestr/c13466 rt2.pdf.

14. (a) Fischer E. Ber $1893 ; 26: 2400-12$. b. Fischer $E$, Helferich B. Justus Liebigs Ann. Chem. $1911 ; 27: 2478-86$.

15. MILIUS A, BRANCQ B. Alkylpolyglycosides : les nouvelles orientations. $\mathrm{OCL} ; 2$ (3) : 177-82.

16. MILIUS A, BOITEUX IP, ROLLAND H, TABACCHI G. Nouveaux dérivés de polyxylosides, procédé pour leur préparation, composition en comportant et utilisation comme agents tensioactifs. FR 2807435, 2000. 
17. BARBEITO C, POLOVSKY S, KREEGER L. Fatty acid esters of ethoxylated alkylglucosides. WO 2004 ; 2004(000862) : A1.

18. DESAI NB. Cosmet Toiletries $1990 ; 105: 99$.

19. CHRISTIAN D, FITREMANN J, BOUCHU A, QUENEAU Y. Preparation of amphiphilic sucrose carbamates by reaction with alkyl isocyanates in water alcohol mixtures. Tetrahedron Lett 2004 ; 45 : 583-6.

20. STEVENS CV, MERIGGI A, BOOTEN K. Tensio active glucoside urethane. US 2003 ; 2003/0125482 : A1.
21. STEVENS CV, BOOTEN K, LAQUIERE IMA, DAENEKINDT L. (2003), " Surface active alkylurethanes of fructans », US 6534647 B1, 2003.

22. SCHOTTEN C. Ber $1884 ; 17: 2544$.

23. MARAMALDI G. ESPOSITO MA. Potassium azeloyl diglycinate : A multifunctional skin lightener. Cosmetics \& Toiletries 2002; 117(3): 43-50.

24. BOTH S, FIEG G, REUTER E, BARTSCHICK F, GUTSCHE B. Method for producing Guerbet alcohols. US $2005 ; 6911567$ : B2.
25. PÉREZ L, PINAZO A, ROSEN M], et al. Surface activity properties at equilibrium of novel gemini cationic amphiphilic compounds from arginine, Bis(Args). Langmuir 1998; 14(9): 2307-15.

26. ROGERS RG, BARRESI FW. Malto oligosaccharides derived glycosides. US 2002; 2002/ 0099185 : A1.

27. BAUMANN E. Ber $1886 ; 19: 3218$.

28. RIGANO L, CUCCHIARA M. Azeloyl glycine : A new active in skin disequilibrium. J Appl Cosmetol 2003 : 177-88. 